\title{
Les bergers de Le Clézio: Messages et significations
}

\section{ESJ Humanities}

\section{Majida Sayegh}

Université Libanaise

Faculté des Lettres et des Sciences

Humaines (Section V)

Département de Langue et Littérature

Françaises, Liban

Submitted: 9 September 2020

Accepted: 27 October 2020

Published: 30 November 2020

Corresponding author:

Majida Sayegh

DOI: $10.19044 /$ esj.2020.v16n32p30

(c) Copyright 2020 Sayegh, M.

Distributed under Creative Commons

BY-NC-ND 4.0 OPEN ACCESS

\begin{abstract}
Gaspar et les bergers, allégories de deux mondes différents, la sédentarité et le nomadisme, qui se réunissent par hasard dans un désert émaillé de mirages et de réalités dramatiques. Leur heureuse rencontre se distingue par l'expérimentation d'une nouvelle façon d'échanger qui va au-delà du langage : c'est la communication silencieuse guidée par des sensations auditives et visuelles favorisées par la nature désertique des lieux. Dans un dialogue implicite à travers leur mode de vie, leurs voix, leur musique et leur dance, les bergers réussissent à transmettre à Gaspar le goût de la culture nomade dans sa dimension à la fois réelle et imaginaire. L'enfant fugueur parvient à devenir un membre actif de cette communauté pastorale en accomplissant ses actes et en adoptant sa façon de communiquer. Néanmoins, s'il a acquis certaines de ses compétences, il n'a pas pu comprendre l'acuité de la lutte pour la survie dans le désert qui pourrait sacrifier l'ibis, l'oiseau blanc qu'il a beaucoup aimé en l'appelant «le roi de Genna». Ainsi la rupture est consommée sous le poids infernal de la sécheresse et de la faim. Pourtant, l'escapade du jeune citadin aventureux en plein désert se révèle fructueuse au niveau de l'expérience humaine: il découvre l'essence de la vie et sa valeur authentique en goûtant, à la fois, sa beauté et ses misères. Riche de son expérience chez les bergers nomades, Gaspar regagne sa ville en devenant plus conscient de son humanité et des dangers qui guettent la nature et toutes les créatures vivantes.
\end{abstract}

Mots-clés : désert, enfance, communication, cultures, animaux, survie, nature. 


\title{
The Shepherds of Le Clézio: Messages and Meanings
}

\author{
Majida Sayegh \\ Lebanese University, Faculty of Letters and Human Sciences (Branch V) \\ Department of French Language and Literature, Lebanon
}

DOI: 10.19044/esj.2020.v16n32p30

\begin{abstract}
Gaspard and the shepherds, metaphors for two different worlds, the sedentary and nomadic lifestyles, that chance united in a desert filled with mirages and dramatic realities. Their happy encounter distinguished itself by the experimentation with a new way of communication, one that transcends language. It is a silent exchange, guided by auditory and visual sensations promoted by the surrounding landscape. In an implicit dialogue through their way of life, their voices, their music and their dance, the shepherds succeed in transmitting to Gaspar a taste for nomadic culture in both its real and imaginary dimensions. The runaway child manages to become an active member of this pastoral community by completing its actions and adopting its way of communication. Nevertheless, although he acquired some of its skills, he could not understand the harshness of the struggle for survival in the desert. A struggle that could sacrifice the Ibis, the white bird that he adored, so much so that he called it "the king of Genna". Thus the rupture is completed under the infernal weight of drought and hunger. However, the escape of the young and adventurous city dweller into the open desert proved abundant with humane experiences. He discovers the essence of life and its authentic value by confronting both its beauty and its miseries. Rich in his experience with the nomadic shepherds, Gaspard returns to his city while becoming more aware of his humanity and the dangers that prey on nature and all living creatures.
\end{abstract}

Keywords: Desert, childhood, communication, cultures, animals, survival, nature 


\section{Introduction}

Avant l'exploration de la nouvelle "Les bergers" (Le Clézio J.-M. G., 1978), une grande attention s'accorde particulièrement aux visées riches de cet « écrivain de silence» comme l'appelait Holzberg (1976) dans sa thèse. Il a reçu le prix Nobel de littérature en 2008, et il a consacré sa vie à la littérature dans sa dimension universelle en instituant pour une convergence harmonieuse entre les cultures, en rassemblant terre et ciel, en fraternisant blanc et noir, et en dépassant les démarcations des ethnies et des religions. Sa patience inlassable consistait à fonder une littérature sans frontières. L'œuvre entière de Le Clézio, écrivain amoureux de la nature et passionné de l'univers, est une «communion entre les hommes et les femmes avec l'Univers » comme conclut Sueza (2009).

Dans un entretien fait par Boncenne (1978), la vision philosophique de Le Clézio s'exprimait dans un acte de pensée qui consiste à « aimer les arbres, la mer ou la lumière » sans recours à un système d'idées emboité pour imposer un jugement. Pour lui, il suffit d'aimer la nature et suivre son rythme pour découvrir les secrets de la vie.

Les huit nouvelles de son ouvrage "Mondo et autres histoires" cachent beaucoup de symboles, de leçons et d'exhortations et selon Holzberg (1981, p.148) « un univers enfantin apparait comme un générateur d'idées destinées à comprendre l'essence de la vie humaine ». La nouvelle liminaire "Mondo" et la dernière "Les bergers" se distinguent par leurs messages prestigieux au niveau humain. Mondo, fils de la nature, " en écrivant son nom en suivant le sens de l'aiguille d'une montre, il voulait peut-être évoquer implicitement le rythme harmonieux de la vie avec le temps » comme préconise Sayegh (2020c). Sa personnalité symbolisant le monde incite à saisir le concept de l'équilibre dans la nature en vivant selon l'écoulement du temps en préservant le patrimoine des hommes à travers la diversité de leurs cultures. Dans cette perspective, la nouvelle "Les bergers " est étudiée en essayant d'explorer ses multiples richesses : un enfant citadin en fugue, Gaspar, qui s'aventure dans un désert où il rencontre par hasard un groupe de quatre bergers nomades avec qui, il construit momentanément une communauté de cinq enfants appartenant à deux cultures différentes (la ville et le désert) et «qui s'expriment par des onomatopées » (Manijashvili, 2014). « Les Bergers » met en évidence les caractéristiques de la vie désertique chez les nomades qui s'oppose à l'image de la ville leclézienne qui reflète une « surabondance d'impressions visuelles et auditives [...]. Un embouteillage est ainsi comparé à l'enfer, un carrefour devient un lieu d'assassinat, et partout l'homme est agressé par des caméras» (Boulos, 1999), tandis que le désert, patrie des nomades, un monde sans limites où règne une amitié profonde entre le sable et le vent, représente «l'ouverture de notre imaginaire, la mémoire vacillante d'un espace déserté, non balisé par l'homme, d'un horizon 
sans cesse repoussé devant nos yeux» comme indique Vartian (2000). Le désert symbole de la clarté et de l'évidence offre sa beauté à chaque moment malgré sa dureté apparente, et que sa nature simple décrite par Sueza (2009) « n'est pas perçue par des êtres humains qui ont perdu la sensibilité face au dévoilement des mystères dont la Nature fait cadeau à l'Humanité de façon quotidienne $»$.

Une méthode exploratoire et analytique est utilisée pour scruter l'ensemble du texte afin d'en retirer les informations hiérarchisées qui servent à répondre à la problématique du récit comme postulait Sayegh (2020c). Cette étude analytique sera consacrée aux comportements de ces bergers: leurs occupations quotidiennes, leur langage basé sur des cris mystérieux, des sifflements, des mouvements du corps, à leur communication entre eux, avec les animaux et même avec la nature, la terre et le ciel. L'auteur pose la problématique de vivre dans un milieu aride qui est privé du strict minimum pour la vie des hommes. Ces enfants bergers isolés du reste du monde est un exemple vivant de la lutte pour la survie et la sauvegarde de leur culture. En effet, s'ils insistent pour y rester, c'est en raison de leur attachement farouche à ce mode de vie et à ces lieux qu'ils connaissent parfaitement grâce à « la plante de leurs pieds ». Ils savaient les bruits du jour et de la nuit, les oiseaux et leurs nids, et les aboiements des chiens et tous les sentiers qui menaient aux endroits où poussaient les espèces végétales. Les enfants avaient établi des liens forts avec leur milieu naturel, une nécessité fondamentale pour leur conservation et celle de leurs bêtes. Malheureusement ces liens « semblent s'être distendus en raison de la spécialisation des savoirs » comme résume Bouvet (2010).

Cette recherche suit une approche interculturelle qui émerge à la confluence de deux cultures différentes : celle de la ville avec ses modes de vie, et celle des nomades avec ses difficultés de survie dans le désert. Le papier éclaircira l'interaction de Gaspard avec les quatre bergers et leur troupeau, et même avec ce qu'ils regardaient dans le ciel étoilé qui miroitait leur monde terrestre. Comment se déroule-t-elle l'interférence entre les deux cultures ? Les cinq enfants réussissent-ils à se comprendre ? Quels types de sons et de mots utilisent-ils pour communiquer et expliquer leurs actions et celles des créatures avec lesquelles ils coexistent ? La diversité culturelle enrichira-t-elle les deux parties ? Quel genre d'enseignements Gaspar peut-il recevoir dans l'immense école du désert?

Cet article tente de saisir ce langage particulier adopté par le groupe des bergers pour communiquer entre eux ainsi qu'avec le milieu extérieur. Le but est de saisir les enseignements qui émanent de cette expérience culturelle pour fonder, peut-être, une vision qui consiste à bâtir une civilisation basée sur le respect de la nature, la cause de notre existence. 
Le corps de l'article est divisé en deux sections, chacune d'elles est subdivisée en deux sous sections. La première section va étudier, d'un côté, la communication entre les enfants et les éléments naturels, et de l'autre côté, les enseignements prestigieux de la nature. La deuxième section consiste à déceler l'essence de la coopération entre les enfants pour vivre harmonieusement en conciliant l'individu et le groupe. Elle est à son tour formée de deux sous sections : la coopération pour vivre son bonheur lors de la fertilité de la nature, et la complicité pendant la saison de la sécheresse et le conflit qui peut en surgir. L'article se termine par une conclusion.

\section{L'habileté de communication des bergers et les enseignements passionnants de la nature}

Les bergers forment un groupe de quatre enfants qui se sont rencontrés par hasard, là-bas devant le mur de pierres sèches, avec Gaspar, un garçon qui avait fugué de sa ville. Le style de Le Clézio nous présente des personnages « dont la première particularité est la différence » (Ben Aïssa, 2013). Que se passe-t-il lorsque se rencontrent deux cultures différentes ? La culture de la ville qui veut dire, limitations et contraintes quotidiennes, et la culture du désert, où domine la liberté de mouvement associée à une variation de température entre le jour et la nuit qui contraste vivement avec l'uniformité et l'infini de l'espace sablonneux. Les enfants parlaient un langage très particulier que Gaspar ne comprenait pas. Comment ce garçon pourrait-il communiquer avec eux ? Aussi comment les enfants devraient-ils procéder pour lui faire comprendre leur lexique ? Les réponses à ces questions seront possibles si un cheminement revient d'abord au rôle qui incombe au langage selon Le Clézio : "le langage ne représente qu'un des moyens à notre disposition pour sonder les mystères du monde » comme énonçait Vogl (2005).

\subsection{Communication particulière entre les enfants}

Les enfants vivaient à l'état presque primitif, c'était d'abord la région des dunes dispersées dans le désert, puis le pays de "Genna", le paradis en langue arabe. Vivre dans le désert nécessite une adaptation avec les échelles du temps et de l'espace. Contrairement à ce qu'il y avait dans la ville de Gaspar où le mode de vie est accablé par les routines quotidiennes et le bruit omniprésent, le monde désertique est à l'écoute d'un mélange subtil de voix diversifiées et naturelles : le crissement de vent sur les feuilles mortes, les cris et les voix des enfants bergers, l'aboiement de chiens sauvages, le bêlements de chèvres et de moutons, le coassement de grenouilles, le vrombissement de mouches, le sifflement de serpents, le glapissement de 
renards, etc. Dans ce monde, ces bruits générés par les différentes créatures formaient « un grincement continu qui résonnait dans le ciel (248)» comme pour traduire l'écho vivant d'une existence naturelle commandée par les éléments. Même l'air participait à cet orchestre naturel en faisant un bruit étrange en déplaçant le sable dans des nuages de poussière d'un endroit à un autre.

En entrant dans le désert, il fallait tenir compte du vent qui effaçait les traces sur le sable, comme s'il empêchait un fugueur de retourner sur ses pas. Dans ce milieu qui brouillait les pistes, le jeune garçon Gaspar arriva en suivant « des sentiers entre les dunes (251)», en quittant la ville, et portant ses habitudes et son langage qui ne correspondaient pas à la vie dans le désert. Il était impressionné quand « il avait entendu tout près de lui, un bruit bizarre (Frrtt ! Frrtt ! (252). Il ne comprenait pas de quoi s'agissait-il exactement, était-il une voix humaine ou animale ? C'est à cet endroit que Gaspar avait rencontré les quatre enfants nomades. Le monde humain et le monde animal se confondaient et résonnaient dans des échos renvoyant la complémentarité qui présidait à leurs existences. D'ailleurs, la reconnaissance entre les bergers et Gaspar passait par la mélodie des sons : même les prénoms se transformaient en musique évocatrice. Il s'appelait Gaspar, disait-il, et les enfants répondaient en riant « Gach $\mathrm{Pa}$ ! Gach $\mathrm{Pa}$ ! (253) ». L'aîné des enfants s'appelait Abel. En réalité, "Gaspar avait entendu son nom plusieurs fois quand les enfants se parlaient (262) ». Il portait une fronde en montrant une petite fille craintive en répétant « Khaf... Khaf...Khaf... (255), alors "Gaspar l'appela ainsi : Khaf (255) ». Cette petite fille qui réagissait par la peur devant la présence étrangère de Gaspar se voyait baptisée par lui Khaf. Ainsi, le langage se voit détourné de son usage initial et prend un aspect allégorique. Concernant les deux jeunes frères, Gaspar pensait qu'ils s'appelaient Augustin et Antoine (274), sans en être certain. Selon Augé (1994, p.61), « le nom identifie l'individu en le rattachant à une filiation ». Chez Le Clézio, les noms reflètent une filiation emblématique qui puise dans l'inconscient collectif et souligne la dimension mythique de ses personnages comme le cas de Mondo où ce prénom devient l'emblème de tout un monde d'épreuves dans la réalité désolante du monde comme le dit Sayegh (2020c). Le prénom d'Abel, l'aîné des bergers est plus qu'évocateur: Abel et Gaspar sont deux garçons de deux cultures différentes, le premier représentait la culture des nomades tandis que le second représentait la culture dite moderne associée à la cité. Ces deux prénoms " nous indiquent par la référence religieuse la possibilité d'une discorde [...]. Gaspar est le nom d'un des trois rois mages dans la tradition chrétienne. Gaspar n'est pas Caïn, c'est Abel qui le devient " comme soutenait Roussel-Gillet (2008). 
Abel, l'aîné, se distinguait par son rôle de chasseur et protecteur du groupe. Son arme elle-même était primitive, élémentaire et remplissait sa fonction à merveille. Manier la fronde nécessitait un savoir-faire acquis avec le temps et l'expérience directe. Il tendait sa fronde (une pièce centrale, réservée pour le projectile qui est généralement un caillou, et attachée à deux lanières tenues à la main) à Gaspar en disant « Goum ! (254) », puis il lui montrait comment s'en servir en répétant «Goum ! Goum ! (254)», mais Gaspar échouait à l'utiliser comme il fallait et « la tresse siffla et cingla son dos, si fort qu'elle déchira sa chemise (254) ». L'échec de Gaspar faisait rire beaucoup les enfants qui criaient «Gach Pa! Gach Paaa! (254) ». Gaspar gêné par son échec voulait leur montrer « son petit miroir (255) » qu'il savait utiliser parfaitement. Ce miroir représentait la trace d'une culture urbaine qui essayait de miroiter la vérité des choses sans la pratiquer réellement. Il captait dedans les rayons du soleil comme s'il voulait maîtriser la nature qui lui échappait. Ce miroir servira comme moyen d'envoyer des messages au reste du groupe. Grâce à un caillou envoyé par Abel avec des cris aigus, Gaspar comprit le message selon lequel il fallait suivre les enfants qui lui criaient « Haa-hou-haa ! (256)» et leur répondit avec le reflet de son miroir. Il était très enthousiaste de les joindre et « se mit à courir de toutes ses forces à travers la plaine (256) ». Il était tellement assoiffé, mais d'où il pouvait-il boire ? Et comment pouvait-il exprimer sa soif aux enfants qui ne comprenaient pas sa langue ? En faisant signe à sa gorge, la petite Khaf lui avait donné une graine poivrée qui l'aidait à humecter sa gorge. Abel lui montrait les graines en disant «Lula (257) ». C'était une indication claire que les bergers s'étaient bien adaptés à cet environnement, eux et leur cheptel.

Quand ils voulaient rassembler les betes, les enfants émettaient de drôles de cris et en agitant leur bras « Hawa! Hahouwa ! [...], Ha ! Hawa ! Hawap ! (258) ». Le troupeau était formé d'une dizaine de moutons et quelques chèvres et l'ensemble était commandé par un bouc noir de longues cornes acérées, qu'Augustin appelait Hatrous. Les chiens répondaient aux sifflements des bergers : «Haw ! Haw ! Haw ! Haw ! (258)». Les quatre "Haw" seraient peut-être la bienvenue adressée aux quatre enfants bergers.

A partir de cet instant-là, un échange commençait entre eux sans interrogations et sans réponses car " c'était un endroit où on ne devait pas poser de questions (256) » parce que l'individu arrivait, peut-être, tout seul, à comprendre en trouvant les réponses adéquates à ses propres questions jaillies du fond de lui-même et de la nature. D'ailleurs, Gaspar s'était rapidement adapté à la vie des quatre bergers en pratiquant leur langage d'onomatopées et tournoyant sa veste comme une fronde en criant aussi « Ha ! Haaa !». Ce comportement nouveau de la part de Gaspar témoignait d'un désir profond de retrouver l'innocence de l'être primitif en essayant 
d'adopter ses manières de s'exprimer. Ainsi, il tentait de «se (re)découvrir en découvrant l'autre, l'animal, [...], la richesse de compréhensions différentes face à cet inconnu » comme écrivait Chanvallon (2010). En effet, ce langage spécial composé de sifflements et d'onomatopées était compris des bergers et servait aussi à communiquer avec les animaux. L'une des compétences exigées du berger pour mener le troupeau était la maîtrise de ces signaux particuliers qui n'obéissaient qu'à l'imitation vocale et la musicalité. Ce langage évitait toute brusquerie surtout quand le berger visait à préserver les animaux serrés entre eux. Toute bête égarée risquait l'attaque des harceleurs, loups ou chiens sauvages. Ce langage associé aux cris et aux sifflements était adressé aussi aux oiseaux et même aux bêtes sauvages comme les serpents.

Après une longue marche, les enfants étaient arrivés à la terre de Genna, une oasis au beau milieu du désert. La beauté de l'endroit se reflétait à travers leurs réactions : Khaf courait en criant «Mouïa-a-a-a ! (266) », et même Gaspar adoptait le même mot avec les autres enfants sachant qu'il pensait au mot "eau". Tout le monde courait vite vers le lac en criant le même mot «Mouïa ! Mouïa-a-a-a ! (266) ». Durant la vie à Genna, la personnalité de Gaspar avait connu un tournant majeur : physiquement le soleil avait brulé son nez, « il perdait sa peau par petites écailles (271) », et la petite Khaf ayant le visage couleur de cuivre, riait quand elle le regardait en disant « Zezay! (272)». Elle le comparait à un « long lézard gris qui perdait sa peau au soleil (272) ». Moralement, sa vie avait pris une nouvelle dimension en changeant radicalement. L'engouement de Gaspar pour la vie à Genna l'avait poussé même à s'occuper parfois d'Hatrous, le bouc noir. Il allait à sa recherche en faisant des onomatopées « Ya-ha-ho ! (278) ». Il scrutait de plus en plus la vallée, à pieds nus, et avec le reflet de son miroir, il saluait de loin les enfants qui, à leur tour, répondaient « ha-hou ha! (286).

Gaspar est devenu actif parmi les enfants en montrant une envie d'apprendre à chasser. A cet effet, Abel « choisit plusieurs lames d'une herbe spéciale, vert sombre, qu'il appelait goum (269) » en faisant une tresse, pour en faire une fronde à Gaspar qui avait travaillé assez dur pour l'achever, et à chaque défaillance, « il devait reprendre depuis le début (269) ». Il lui fallait de l'effort pour bien maîtriser l'usage de la fronde car le caillou lancé risquerait de frapper le lanceur lui-même ou de provoquer un danger pour les autres. Après une démonstration sérieuse de sa solidité par Abel, Gaspar l'essayait sur un tronc d'un palmier en insistant pour toucher avec précision la cible car il considérait que " la fronde faisait partie de lui-même (270) ». Avec cette fabuleuse réussite, Gaspar « sut qu'il n'était plus le même (270) ». Il pouvait chasser et participer à la protection du troupeau avec Abel : deux tâches octroyées exclusivement à ceux qui maitrisaient la vie dans le désert. Gaspar enrichissait le potentiel du groupe des bergers car ils étaient 
devenus « deux à chasser (272)» des lièvres, des perdrix et des oiseaux. Les deux garçons communiquaient à travers les sifflements de leurs frondes en étant fiers de leur grand rôle dans la protection des bergers et leur troupeau.

La nature enseignait à Gaspar le vrai sens de la vie : responsabilisation et participation active à la vie du groupe qui est le secret du bonheur collectif. En réalité, la fronde n'est pas une simple arme que le nomade portait : elle est le moyen polyvalent pour survivre et protéger le troupeau. Ses multiples fonctions soulignent l'acuité de ses rôles dans ce désert car elle servait aussi à envoyer des messages, pour rassembler les bêtes autour d'Hatrous, pour scruter le plateau de la chasse, pour éloigner ou tuer un chien sauvage, pour battre le nuage vivant de sauterelles, pour chasser des lièvres, pour se défendre lors d'un conflit. C'est pourquoi, maîtriser son usage c'est dominer le milieu dans lequel on vivait. Elle est l'emblème du berger nomade et son monde désertique. Avec la fronde Gaspar gagnait la confiance des bergers et leur respect en adoptant leur mode de vie et leur façon de communiquer.

\subsection{Enseignement prestigieux de la nature et des animaux}

Après la maîtrise primordiale de l'arme du désert, la fronde, vient l'apprentissage des signaux envoyés par les éléments naturels. Abel, guide et protecteur du groupe des bergers, suivait attentivement la nature, pour lui, le vent portait des informations importantes qu'il devait prendre en compte pour choisir sa direction dans la prairie car « le vent qui passait sur l'herbe apportait les bruits tenus de la vie, les odeurs (273) ». N'était-ce pas une leçon pour son compagnon Gaspar ? Ce monde apparemment silencieux ne l'était pas en réalité car très chargé de signaux que l'homme doit interpréter pour savoir se conduire dans son milieu. Dans sa dureté, le climat désertique offre la possibilité, pour celui qui y vit, de contempler et d'en tirer les leçons. L'oasis dans sa générosité offre à cet égard une contradiction criante nécessaire pour mettre l'homme en face de la vie dans toutes ses contradictions directes et décisives : la nudité du désert embellit et rend l'espace d'une oasis un véritable paradis pour un nomade assoiffé la plupart du temps.

Vivre en harmonie à Genna, c'est pouvoir communiquer avec le vent, les parfums des fleurs, les balancements des branches de palmiers, les frissons des feuilles de papyrus, surtout avec l'oiseau blanc que Gaspar avait vu au milieu du marécage dont le plumage « faisait une tache éblouissante sur l'eau grise (275) ». L'oiseau se levait, s'éloignait un peu puis il "s'arrêtait et regardait Gaspar (275) » qui souhaitait lui faire comprendre « qu'il voulait être son ami (276) ». Pour Gaspar, il n'était autre que « le roi de Genna (277)». Il s'engouait de plus en plus de la nature et de la façon dont les bergers y vivent. Il accompagnait Augustin et Antoine pour relever 
les pièges en apprenant cette technique qui consistait à faire tromper l'animal pour le capturer. Un jour, un petit renard était tombé dans le piège, ils l'avaient donné à Khaf qui l'appelait "Mîm". Elle le protégeait et lui donnait à « boire du lait de chèvre dans le creux de sa main (274)».

Réellement, les animaux jouaient un rôle important dans le quotidien de ces bergers. Hatrous, le bouc au regard mystérieux, était l'ami d'Augustin. Ce garçon calme était le seul à pouvoir s'approcher de lui en chuchotant dans ses oreilles ses belles paroles chantantes qui poussaient Hatrous à s'arrêter "de mastiquer l'herbe pour écouter la voix du petit garçon (279) ». C'était un animal qui laisse croire qu'il « savait tellement de choses, non pas de ces choses qu'on trouve dans les livres, dont les hommes aiment parler, mais des choses silencieuses et fortes, des choses pleines de beauté et de mystère (279) ». Il connaissait les pâturages dans le désert, dans la plaine, toutes les herbes et les arbustes qui y poussaient. Il distinguait entre les différents sentiers et diagnostiquait avec précision les traces de renards et de serpents. Il était le chef préoccupé des membres de son troupeau. Dès qu'il avançait parmi eux, les chèvres et les moutons dansaient en exprimant leur joie. Sa présence annonçait-elle peut-être un déplacement imminent vers d'autres pâturages plus tendres. Augustin pensait qu'il avait besoin d'une " vie entière » (279) » pour les connaissances mystérieuses d'Hatrous, c'est pourquoi avec sa voix basse, il lui demandait de lui « enseigner des nouvelles choses (284) ». La réponse du vieux bouc ne tarde pas à répondre et en remuant ses mâchoires «il se penchait sur Augustin et soufflait sur son visage [...] Il lui enseignait ce qu'il avait appris dans le désert, les journées sous le soleil qui brûle, les choses de la lumière et de la nuit. Peut-être qu'il lui parlait du croissant de lune suspendu au-dessus de l'horizon, ou bien du grand serpent de la voie lactée qui rampe à travers le ciel (284)». Gaspar qui s'émerveillait devant de telles scènes espérait qu'un jour puisse comprendre lui aussi les précieuses leçons d'Hatrous. Un autre animal était nécessaire au troupeau et qui faisait équipe avec le bouc. Augustin domestiquait un chien sauvage et 1 'appelait "Noun". Comme tout enfant attaché à son chien, il « lui lançait des pierres (280) » en s'enfuyant devant lui, et ceci énervait énormément Hatrous et le rendait jaloux. Le petit berger sifflotait entre les dents de Noun, une sorte de communication entre eux, qui différait de celle qui était pratiquée avec le bouc noir. Noun ne quittait jamais le troupeau, il « restait près d'Hatrous et du troupeau (280) » et les deux formaient une équipe de gardiens.

A la tombée de la nuit, quand les membres du troupeau rentraient serrés les uns contre les autres derrière leur chef taciturne, était le moment préféré de la journée pour Gaspar et Augustin qui contemplaient le fond noir du ciel où les étoiles de Genna "dessinaient des figures étranges qui couvraient le ciel (282) ». Augustin aimait vaguer dans leur éclat en leur 
donnant des noms qu'il avait inventés lui-même comme «Altaïr, Eltanin, Alkaïd (qui signifient respectivement l'oiseau, le dragon et le capitaine en langue arabe), Kochab, Merak, Alderamin, Midrash, ... (282) ». Il leur parlait comme s'ils étaient des amis qu'il avait rencontrés après une longue absence. Les deux compagnons regardaient là-haut où « il y avait beaucoup de monde, beaucoup de peuples allumés, des oiseaux, des serpents, des chemins qui sinuaient entre les villes lumière, des rivières, des ponts; il y avait des animaux inconnus arrêtés, des taureaux, des chiens aux yeux étincelants, des chevaux "Enif" (283) ... ». Il y avait aussi des corbeaux et des géants qui régnaient "couronnés de diamants, immobiles, et qui regardaient la terre, ... (283) ». Mais surtout, c'était le grand bouc noir Hatrous, "debout dans la nuit, qui régnait sur son univers "Ras Alhague" (283) ». En réalité, il voyait dans Hatrous comme étant le chef qui régnait au sommet de la vérité car (Ras Alhague) veut dire " la tête de la justice et son essence. Ainsi, pour Augustin, le ciel est un tout magique. C'est-ce que Roussel-Gillet (2008) a validé en disant «Le ciel ouvre un espace d'infinitude, qui invite à la lévitation » et selon Tristmans (2005) « le ciel étoilé n'offre guère de stabilité, mais se fragmente et se fait miroir de la violence des hommes ».

L'image d'Hatrous sur terre en tant que leader marchant au milieu des bêtes peut être un reflet de son image dans le ciel, c'est comme s'il conduisait son troupeau d'étoiles rassemblées autour de lui. C'est une belle image qui relie le ciel à la terre. En face de cette incroyable aventure, Augustin qui fixait pleinement son attention sur les étoiles pour « entendre le murmure léger qui résonnait au fond du ciel, le bruit d'eau et de feu des lumières lointaines (283) » parlait du ciel comme s'il avait un savoir astral qu'on ne le voit pas ailleurs et il se manifeste comme un "réparateur de continuité " selon l'expression de Tristmans. Au lieu d'être écrasé par le ciel et subissant passivement ses caprices, le nomade réinvente le peuple du ciel et le fait à son image et non le contraire. De cette façon, Hatrous devient le maitre là-haut comme il l'est sur terre avec son troupeau. Le bédouin semble maîtriser davantage sa vie et son imagination qui est au service de son bonheur et son harmonie avec l'existence, tandis que l'imaginaire déformé du civilisé l'a détrompé et l'a fait plonger dans les désillusions et devient ainsi plus malheureux.

Les techniques modernes n'avaient pas de place dans l'imaginaire bédouin qui ne ressentait pas le besoin d'en avoir: leur soif va vers les produits de la nature pour combler leurs besoins vitaux. Aller au-delà de la nature ne les tentait pas, car la perfection pour eux c'était elle. Quand ils voyaient un avion dont "le sillage fendait le ciel en deux (290) » ils se demandaient si cet avion apprenait «des choses que ne savent pas les oiseaux (290) ». Une façon de s'interroger sur les bienfaits de la technologie 
en comparaison avec les bienfaits naturels. Clin d'œil aussi à la primauté de tout ce qui est dans la nature (éléments et créatures) et son utilité véritable pour l'équilibre dans la vie des hommes. Pour eux, un oiseau pouvait résumer à lui seul toutes les aspirations humaines pour le vol. Ses ailes déployées dans le ciel véhiculaient avec elles le sentiment de la liberté dans un espace sans frontières. Ils s'inspiraient de tout ce qui les entourait et chaque être vivant pouvait être pour eux un enseignant. Ils s'étaient parfaitement familiarisés avec toutes les créatures et même le renard Mîm que Khaf élevait, préfigurait l'arrivée des sauterelles dans la région en donnant des signes particuliers à l'aide de ses oreilles attentives et son corps tremblant « C'est Mîm qui les entendit le premier, bien avant qu'elles aient apparu au-dessus de la vallée (292) ». Chaque être vivant avait une place et un rôle. Gaspar, le nouvel arrivant l'avait remarqué. A Genna, les enseignants sont partout « il y avait beaucoup de choses à apprendre (290) » et « Gaspar savait bien que les gens d'ailleurs ne pouvaient pas les apprendre (291) ». Seuls les bergers nomades les détenaient grâce à leur vie dans le désert. Leurs livres ne ressemblaient pas à ceux de la ville : les connaissances étaient cachées dans les éléments de la nature, le ciel et ses étoiles, la chaleur du jour et le froid de la nuit désertique, la soif et la rareté de l'eau, les arbres et leurs ombres, les oiseaux de Genna, et surtout les animaux familiers et sauvages qui accompagnaient les bergers. Les enseignants étaient partout dans le désert, dans les grains de sable et leur infini, et même dans les insectes qui s'y cachaient. L'exemple des villes de termites on était un grand exemple : Gaspar se sentait proche des insectes «C'était un peu comme s'il était leur semblable [...]. Il pensait qu'elles aussi [les fourmis] avaient des choses à enseigner » (287). Les « grands immeubles aveugles (288)» de ces insectes lui donnaient l'envie de construire des villes aussi superbes mais avec fenêtres ouvertes à toutes les diversités du monde. En effet, Gaspar pensait que s'il pouvait acquérir tous ces enseignements, il deviendrait comme Hatrous, un grand inspecteur du paysage qui construirait «des grandes maisons de boue, [...] avec juste une fenêtre au sommet, d'où on verrait toute la vallée de Genna (292) ». Pour mieux apprendre, il ne faut pas être dans une salle de classe, prisonnier d'un siège et d'une table, mais comme disait Gaspar, « il fallait être à Genna, avec les bergers, avec le grand bouc Hatrous, le chien Noun, le renard Mîm, avec toutes les étoiles audessus de vous... (291) ». Cela rappelle la façon dont Mondo a appris à lire et à écrire. Dans cette école pas comme les autres où l'apprentissage prend la forme d'un échange chaleureux avec une nature aimante et aimée comme évoque Sayegh (2020c). La connaissance s'avère un jeu comme l'apprentissage de Mondo. Le ludique est constructif et presque mythique. Ce que faisait Khaf est fortement informatif : "elle fabriquait avec de la boue des assiettes et des plats, qu'elle mettait à sécher sur les feuilles. Elle 
faisait aussi des sortes des poupées, qu'elle coiffait de brins d'herbes... (291) ». Créer son propre monde commence par façonner ses choses imaginaires : mettre la main à la pâte s'avère un moyen efficace pour donner forme à ses rêves. N'est-ce pas fait par l'enfant Alexis dans (Le Chercheur d'or) de Le Clézio (1985) où il fabriquait, comme khaf, un navire de boue pour sauver le Boucan familial et l'emporter loin de tout danger?

\section{La nécessite de coopérer pour vivre}

La vie dans le désert diffère verticalement de celle en ville pleine de contraintes et de bruits gênants. En tant que nouvel arrivant, Gaspar explorait cet environnement désertique totalement distinct de son milieu habituel et déployait une grande énergie pour s'intégrer à ce monde nomade dont il ne comprenait ni la langue «ni les codes régissant l'ensemble de ses pratiques culturelles » comme expliquait Bouvet (2012). Face à cette altérité il se comportait positivement en respectant la particularité culturelle de l'autre et loin de la considérer comme une barbarie, il savait la transformer en une richesse capable de construire. C'est ce qu'affirme Diouf (2016) «l'identité n'est pas une donnée figée chez Le Clézio. Elle procède d'un processus qui combine deux mouvements rhizomiques qui ne sont contradictoires qu'en apparence : déconstruire pour mieux reconstruire ».

En effet, malgré la cruauté de la vie dans le désert, la rareté de l'eau et de la nourriture, des avantages et des richesses insoupçonnées se cachaient partout. Chaque rocher de la vallée abritait un secret de la vie naturelle. Le désert est la mémoire des hommes qui y ont vécu depuis longtemps. Il est " l'ouverture de notre imaginaire, la mémoire vacillante d'un espace déserté, non balisé par l'homme, d'un horizon sans cesse repoussé devant nos yeux» comme résumait Vartian (2000). Avec ses dunes qui se déplacent perpétuellement au gré du vent, il ressemble à une mer avec des vagues de sable auxquelles répond l'infinité du ciel étoilé du soir. Entre ces deux espaces infinis, les bergers nomades passaient leur temps de jour comme de nuit.

Dans son livre (Désert), Le Clézio (1980) illustre une vision propre des nomades, où chaque individu vit comme un fils du sable, du vent et de la liberté, doué d'une capacité d'adaptation illimitée. C'est pourquoi, ils peuplaient ces espaces de leurs formes familières comme pour répondre à leurs immensités effrayantes.

Mais le désert n'est pas seulement un espace vide peuplé par l'imagination du nomade, il possède des endroits très fertiles et verdoyants comme « la plaine d'herbe et d'eau. Légère, douce, délicate comme la mer vue de loin (260) ». La beauté se manifestait avec obstination dans les hauts arbres avec leurs branches qui se balançaient avec l'air. A cet endroit plein 
de vitalité chaque être vivant semblait chercher à se préserver jalousement dans un milieu où la nature se nourrissait d'elle-même.

Afin de saisir les deux faces de l'aventure de Gaspar dans ce milieu désertique, il serait très utile de diviser cette section en deux parties : la première est liée à la période de la fertilité marquée par la présence de l'eau du lac de Genna, pivot de l'histoire, qui était la raison d'une grande satisfaction pour les enfants et pour le troupeau. Tandis que la seconde sera consacrée à l'autre versant de la vie de l'ensemble, enfants et bêtes, pendant la sécheresse où l'eau du lac s'épuisera et devient non potable.

\subsection{La vie à Genna : Coopération, complémentarité et bonheur}

L'eau du lac était une source de plaisir pour les enfants et leur troupeau. Même pour le reste des animaux et surtout les oiseaux qui buvaient les gouttes d'eau en chantant et en rafraichissant leurs ailes en voguant doucement à la surface du lac. L'annonce de cette eau transfigure l'aspect maussade des bergers nomades : l'air farouche de Khaf avait été changé lorsqu'elle s'était aperçue de loin le pays de la verdure et de l'eau. Elle devenait plus détendue en dressant sa main pour montrer à Gaspar la vallée miroitée près de l'horizon, en disant « Genna (261) ».

En réalité, avant d'arriver à cet endroit paradisiaque, les bergers vivaient en plein désert, un milieu qui nécessitait une grande endurance pour résister aux fatigues physiques et psychologiques, c'est pourquoi ils étaient si assoiffés d'une oasis qui pouvait combler leurs désirs. Cette alternance entre l'aridité et la profusion donnait à leur vie nomade un aspect changeant et diversifié. Ainsi, l'altérité enrichissante s'exprimait dans cette complémentarité entre l'infini du désert et l'insularité de l'oasis. A l'immensité du désert, répondait la réduction rassurante de Genna. C'était comme une halte au milieu d'une course interminable entre la vie et la mort. Les enfants et leur troupeau se sentaient chez eux à Genna, " c'était leur terre (265) ». C'est une plaine entourée de collines, riche de toutes sortes d'arbres, comme «des amandiers, des peupliers, des lauriers géants, [...], des hauts palmiers (265) ». La verdure envahissait le milieu, la beauté de la nature s'étendait dans toutes les directions, et au milieu se situait un lac doux enveloppé de palmiers et scintillant comme un miroir. La profusion de la vie à cet endroit évoque l'idée de l'Eden chez Le Clézio qui croit en la présence d'un possible paradis terrestre. En effet, dans un entretien particulier que Dutton (1999) avait réalisé avec lui à Paris, le 29 mai 1997, et à la question «Le paradis n'est pas ailleurs?», il avait répondu : « Le paradis n'est pas ailleurs, mais c'est maintenant. Je crois qu'il faut tendre à réaliser le paradis sur terre, c'est-à-dire enfin réaliser l'utopie ». Pour les bergers, le paradis ressemblait tout simplement à une oasis au milieu du désert. 
A Genna, chacun exprimait sa propre joie, en poussant des cris croissants. Le plus fort des enfants s'occupait du plus jeune. Gaspar par exemple, portait sur ses épaules la petite Khaf parce qu'elle ne pouvait pas accélérer dans ses marches à cause des longues herbes qui «cinglaient ses mains et son visage (266) », mais c'était elle qui choisissait le chemin le plus court pour être "dans la direction de l'eau (267) ». L'eau était vitale et génératrice de sécurité pour les enfants. En arrivant au lac, tout le monde jouissait en s'abreuvant goulûment, puis les enfants s'acheminaient vers la détente sur les rives du lac.

Pour affirmer qu'ils s'accrochaient tellement au lieu, les enfants décidaient de construire une petite hutte, « une sorte d'igloo en terre, avec un côté ouvert pour entrer (268) ». C'était un travail d'équipe par excellence. Tout le monde avait contribué à son achèvement et chacun selon sa capacité : Abel qui était l'architecte «avait coupé de longs roseaux et des branches (267) » et avec Gaspar et Antoine, il « avait formé la carcasse (267) », Khaf et son frère Augustin ont été chargés de préparer la boue pour l'étaler sur les murs « en tapotant avec les paumes de la main (267) ». Les roseaux et les branches étaient la base de cette maison primitive dont les interstices des murs étaient bouchés « avec des petites herbes (267)», tandis que le toit était composé de feuilles de palmiers. Le bien-être semblait toucher les enfants et leur troupeau. Ils avaient maintenant tout ce qu'ils espéraient : un toit, de l'eau potable, des pâturages verts, de la nourriture assurée à travers le lait de chèvres et la chasse. En plus de tout cela, la sérénité dominait à Genna, le ciel était si clair et il attirait paisiblement l'attention et « on pouvait rester des heures à regarder le ciel, sans rien faire d'autre (268)».

La chasse était primordiale pour compléter le lait des chèvres. Abel avait donné un exemple dans sa chasse aux lièvres en utilisant sa fronde. Quand il réussissait à en capturer un, « ensemble les enfants coururent pour le ramasser (264) », puis, une fois nettoyé, ils l'embrochaient sur une branche pour le dîner, non seulement pour eux et Gaspar, mais aussi pour les chiens sauvages. Abel offrait d'abord le repas à Gaspar car il était traité comme un hôte, puis pour lui et ses frères qui mangeaient rapidement et jetaient les os aux « chiens sauvages (264) » qui attendaient à leur côté. Cette image de vivre en famille très solidaire a été clairement manifeste dans leurs habitudes lors de la préparation du repas, et dans leur attitude envers des chiens. En effet, ces chiens sauvages circulaient dans toutes les directions en cherchant à se nourrir et ils accompagnaient les enfants et leur troupeau dans leurs déplacements en s'accrochant à eux. Et quand le troupeau rentre au campement, ils marchaient derrière eux en attendant des os éventuels. Pendant l'absence des enfants, les chiens sauvages n'attaquaient pas le troupeau, soit par peur des représailles du bouc noir Hastour qui le surveillait, soit parce qu'ils espéraient quelques nourritures. Ainsi, un 
équilibre spontané et simple régnait entre les deux espèces : humaine et animale.

Cet équilibre naturel se voit même avec les insectes qui surgissaient de l'intérieur de la terre: l'exploration des termitières se révélait une occasion de plus pour découvrir et apprendre de ces espèces souvent méprisées par les humains. Les fourmis et les scorpions n'étaient point moins enseignants que les autres espèces. En effet, pendant l'exploration du pays des termites, "Abel regardait, penché en arrière, prêt de s'enfuir : mais Gaspar avançait déjà le long des rues, au milieu des hautes tours, en balançant sa fronde le long de sa jambe (288) ». Pendant cette exploration du pays des termites, Gaspar, assoiffé de découvertes, semblait plus aventurier et plus offensif qu'Abel. Son courage et son intelligence le rendaient utile au groupe de bergers. Son ingéniosité dans l'utilisation de la fronde, avait sauvé Abel d'un chien sauvage quand il s'était battu avec lui pour sauver une chèvre égarée. C'était la première fois qu'Abel demandait le secours de son compagnon après avoir raté le chien sauvage. Gaspar « chargea sa fronde avec une pierre aiguë et la fit tourner de toutes ses forces (271) », il avait réussi à frapper et briser le crâne de l'animal.

L'intégration de Gaspar dans la vie nomade semble totale. Vivant intensément le quotidien des bergers, il «ne se souvenait plus très bien de ce qu'il avait connu avant qu'ils arrivent ici, à Genna (271) ». Quand la ville lui venait à l'esprit, il imaginait des rues, des voitures, des camions, des avertisseurs sonores, des klaxons, de la pollution due à l'échappement de véhicules ce qui contrastait fortement avec le paysage de Genna et sa plaine mystérieuse où le silence et la clarté inondaient les arbres et les herbes qui ondulaient au gré de la brise. Dans ce milieu édénique, les enfants bergers exprimaient leur joie à travers la musique et la danse : un peu avant le coucher du soleil, Khaf dansait au rythme de la flute sur lequel « Augustin et Antoine jouaient à tour de rôle (289) ». C'étaient des notes, comme des cris d'oiseaux qui émerveillaient Gaspar. La danse de Khaf était caractérisée par la régularité et l'harmonie des mouvements de son corps, «le buste bien droit, les mains écartées le long de son corps, elle frappa le sol avec ses pieds nus, d'un mouvement rapide de la plante de pied et des talons, (290)». Cette danse du serpent continuait jusqu'au coucher du soleil. Augustin partait pour rejoindre le troupeau mais « il continuait à jouer tout seul là-bas (290)». Dans cette ambiance de bonheur et de sérénité, Gaspar renforçait ses liens avec le groupe d'enfants dont il est devenu une partie intégrante: Khaf demandait parfois à Gaspar de lui imiter « le bruit des autos (271)». Elle était devenue très attachée à lui car elle appréciait les qualités rassurantes de ce doux garçon qui l'aidait à traire les chèvres et plumer les oiseaux. Elle préférait toujours dormir à son côté en lui serrant très fort la main. Ainsi, après avoir réussi à s'imposer comme un membre actif et utile grâce à sa 
fronde, Gaspar s'affirmait dans le domaine psychologique comme étant une source d'affection et de bonheur pour les autres. La vie à Genna avait réussi à faire sortir le meilleur de ce garçon citadin qui ignorait la beauté de la nature et la valeur positive de la chaleur humaine.

\subsection{La vie à Genna : Sécheresse et conflit}

La quiétude des enfants bergers n'avait pas beaucoup duré car la sécheresse est arrivée. Quand la nourriture devint rare, leur ultime espoir était de survivre avec leur troupeau. Le rôle d'Abel et de Gaspar devenait essentiel. Leur coopération s'avère plus que primordial car la faim menaçait toutes les créatures vivantes autour d'eux. Les premiers signes de l'aridité étaient l'arrivée des nuages de sauterelles favorisés par la chaleur brûlante du soleil. Les bergers s'en allaient à la bataille en partageant les tâches : Gaspar et Abel allumaient le feu et « couraient au-devant du nuage, en faisant siffler les lanières de leurs frondes [...], le troupeau fuyait vers les dunes (293)». Hatrous tempêtait manifestement en restant derrière. Les frondes des deux garçons avaient une fonction déterminante avec le feu allumé pour combattre le fléau. Quand quelqu'un d'eux réussissait à frapper le nuage vivant, il criait de joie, l'autre répondait avec un cri d'admiration. Et ainsi la bataille continuait, jusqu'à la disparition des nuages d'insectes dans les collines de pierres. Et finalement, ils rentraient épuisés à leur cabane. Les plus jeunes enfants ramassaient des sauterelles assommées pour leur diner. Le festin n'était pas seulement pour les enfants, mais aussi pour les chiens sauvages.

Abel, l'enfant aîné, avait un devoir de protéger ses frères et sa petite sœur et il était inquiet surtout quand il retournait de la chasse «avec des maigres petits oiseaux aux plumes souillées qui ne calmaient pas la faim (295) ». Que fallait-il faire ? Où fallait-il aller chercher de la nourriture ? La situation était devenue alarmante. La lune revenait pour baigner de ses rayons "la vallée de Genna (295) », mais on sentait un changement « quelque chose d'étrange dans sa lumière pourtant (295) ». Un mélange de froid et de silence dominait dans la région. Les enfants qui appréciaient bien le climat de Genna, "se couchaient tôt dans la maison (295) ». Le plus marquant était la profonde angoisse dans le regard d'Abel qui " partait en balançant sa fronde d'herbe le long de sa cuisse (295)». Les grognements et les jappements des chiens sauvages qui « rôdaient tout le temps à travers la plaine éclairée (296) » témoignaient de la dégradation de leur condition de survie et exprimaient leurs regrets de ne plus obtenir des entrailles que Khaf leur jetait pendant la période de prospérité.

Abel, expérimenté et décideur, connaissait très bien les lieux de la vallée de Genna. Il savait où il pouvait trouver le serpent Nach et l'ibis, l'oiseau blanc que Gaspar avait pris pour ami et l'avait appelé « le roi de Genna ». Pour cela, Abel décida d'aller avant tout chasser le serpent aux 
anneaux qui vivait dans cette région. Les deux garçons partaient ensemble en longeant le marécage « dans la direction des collines de pierre (296) » où se trouvait l'abri de Nach. Gaspar avait déjà entendu le bruit due à son glissement « quand il avait franchi le mur de pierres sur le chemin de Genna (297) ». Il se souvenait de la dance de serpent, que Khaf lui avait montrée un jour, en criant « Nach! Nach! Nach! Nach! Nach! (297)» et en imitant le bruit de crécelle provoqué par sa queue contre les pierres. Khaf avait répété cinq fois son nom, peut-être en l'honneur de Gaspar le cinquième enfant. Abel, imité par Gaspar, marchait à petits pas, silencieux et très attentif. Dans cette nuit froide, Nach cherchait une proie et son objectif était le troupeau. Ils « marchaient avec des gestes ralentis au milieu du pays et de la poussière (298) », en inspectant scrupuleusement le terrain dans le pays de Nach " près d'un acacia brulé par la sècheresse et le froid (298) ». Gaspar entendait le bruit du froissement, le « Frrrtt-frrrtt» qui l'avait entendu un jour quand il était arrivé devant le mur de pierres sèches. C'était Nach au-dessus de sa tête.

Abel savait bien les différentes phases qu'il fallait suivre pour chasser Nach : choisir le temps adéquat, amener une longue branche, garder son silence, savoir faire des sifflets bien étudiés, maitriser la danse de Nach et enfin choisir le bon moment pour lui briser la nuque à l'aide d'un bâton. En effet, Gaspar apprenait que cette musique continue exécutée par Abel avait pour but de garder le serpent à sa place et l'empêcher de s'en aller en pénétrant dans son corps, sous la forme d'ordres, ralentissant son cœur, influençant ses mouvements. Au moment où les glandes de Nach étaient gonflées de venin, la danse forte des garçons devait continuer pour le paralyser. C'est au moment où Nach levait sa tête, Abel cessait de siffler et il s'approchait de lui avec son bâton. Nach redressait « sa tête en angle droit avec son cou (302) » en regardant cette silhouette blanche, et " son corps s'étendit dans toute sa beauté, étincelant comme une armure et moiré comme du zinc (302) ». Mais Abel, avec un seul coup, lui brisa la nuque.

Les enfants, le troupeau et les chiens sauvages devenaient les seuls à Genna. Avec le lait des chèvres, ils déterraient les racines pour calmer un peu leur faim, et Khaf « faisait à nouveau provision de graines poivrées pour le départ (303) ». La colère frappait aussi le troupeau et en particulier Hatrous. Gaspar pensait tellement à ce changement et « il restait allongé sur le dos toute la nuit, près de Khaf (304) ", qui, à son tour, ne dormait pas non plus en serrant Mîm contre elle. Tout était changé à Genna. La nuit si froide faisait serrer les enfants les uns contre les autres et la chaleur du jour est devenue insupportable. Les animaux sont partis à l'exception des scorpions et des fourmis qui se cachaient sous les pierres. Le goût de l'eau du lac devenait non potable et très préjudiciable aux enfants et au troupeau. Les enfants subissaient les conséquences de cette situation électrique. Ils ne 
pouvaient plus assurer leur repas comme avant car les lièvres et les oiseaux avaient fui Genna.

Abel, le responsable des enfants passait son temps, l'esprit dissipé, ses yeux tournés vers les collines. Où fallait-il chercher ? Au fond de soi, il pensait à l'ibis, le seul oiseau qui restait en cachette là-bas au marécage. Il partait à visage tendu, portant sa fronde et sans hésiter, Gaspar le suivait « bien qu'il ne le lui ait pas demandé (305) ». Il s'enfonçait seul dans la boue du marécage en cherchant l'ibis que Gaspar aimait beaucoup : "L'oiseau blanc était bien tel qu'il l'avait vu la première fois (305) ». Gaspar avait toute confiance en lui, en croyant qu'il " gouvernait silencieusement la vallée, les herbes, les collines et les dunes, jusqu'à l'horizon (305) » et qu'il lui suffisait de donner un petit ordre pour que la vie reprenne sa beauté à Genna. Quand Abel faisait tourner sa longue lanière, Gaspar se jetait vite dans la boue « en bousculant les tiges des papyrus (306)». Les deux enfants bataillaient dans la boue, et «l'ibis blanc frappait l'air de ses ailes et prenait son envol (306) ». La chose inattendue était arrivée. Abel faisait tournoyer sa fronde pour frapper la tête de Gaspar qui se baissait et le caillou frappait son épaule, puis un deuxième caillou sifflait près de sa tête. Gaspar avait perdu sa fronde dans le marécage, et il courait plus vite pour échapper à Abel. « La colère, la peur, et la douleur faisaient comme un grand bruit dans sa tête (306) », et « il se sentait triste et fatigué (306) ». Il savait que quitter Genna était inévitable et il reprit le chemin de retour vers sa ville de départ. Les images des enfants, surtout la petite Khaf, du troupeau et son chef Hatrous, occupaient pleinement sa tête. La tempête de sable l'empêchait de courir en frappant ses mains, son épaule et même la poussière entrait dans sa bouche «il courait pieds nus sur la route (309) ». Les enfants savaient bien qu'il fallait aller ailleurs où il y avait de l'eau nouvelle, des nourritures suffisantes pour eux, et pour leur troupeau. Gaspar ne se voyait pas seul, mais il était avec les enfants, le bouc noir Hatrous, le renard Mîm, et le chien Noun «sans avoir besoin de parole (310) ». En arrivant au bureau de la gendarmerie en disant « je m'appelle Gaspar... Je me suis perdu (310)».

\section{Conclusion}

Dans cette nouvelle consacrée aux bergers, Le Clézio révèle la spécificité de la nature désertique à travers un scénario joué par des enfants qui sont les mieux aptes à refléter avec transparence la réalité de la vie naturelle. La vie de ces enfants bergers illustre la simplicité primitive du désert où l'eau représente l'élément primordial de la survie qui guide une recherche journalière de la nourriture pour eux et leurs bétails. Leur dénuement naturel allège leur quotidien et l'enrichit en même temps car ils deviennent les acteurs actifs de leur sort. Cette responsabilisation fait leur 
bonheur mais aussi leur malheur car à la fin elle fait éclater le conflit entre deux visions de la vie représentées par Abel et Gaspar.

Ces jeunes bergers nomades, vivaient deux situations opposées: d'une part, la vie à Genna, la terre de paradis qui les submergeait par sa beauté naturelle couvrant la terre et le ciel, d'autre part, la sécheresse qui les menaçait et aboutit à une descente aux enfers à la fin de la nouvelle.

Gaspar, le citadin, surgit par hasard dans ce groupe et se défait du fardeau de la civilisation pour se mettre à une nouvelle école universelle : la nature. Il refait en quelque sorte son éducation déformée par les bruits parasitaires de la ville qui sont remplacés avant tout par le silence et ensuite par les sons harmonieux de la nature : vents, insectes, oiseaux, animaux et toutes sortes de créatures vivantes qui se concordent pour orchestrer une musique vivante et reposante qui mime la vie de la terre. Cela éduque ses sens et lui ouvre la voie aux leçons précieuses de la nature et le renvoie à ses racines primitives coupées par le superficiel et l'industriel. Ce retour aux sources est consolidé par le langage silencieux et mystérieux qui s'instaure avec les animaux familiers et sauvages, habitants premiers de la planète et messagers de la pulsion vitale nécessaire à tout être vivant.

Pourtant, la terre, cette couveuse idéale des êtres vivants, n'est pas isolée du cosmos : les bergers savaient parfaitement agencer leur savoir terrien avec les messages envoyés du ciel sous différentes formes. Leur vie naturelle n'est pas prisonnière du sol et leur imagination est nourrie du vaste ciel illimité du désert. Ainsi, l'infini du sol est complété par l'infini du ciel pour aboutir à une liberté incroyable.

La nouvelle « Les bergers » est avant tout un appel de Le Clézio pour ouvrir les fenêtres des cités modernes aux autres communautés de nature différente pour communiquer et se compléter. Et comme le dit (Dufour, 2018) dans sa thèse qui invite à un dégagement d'une stratégie qui consiste à libérer chaque groupe humain de ses propres frontières en préservant le droit de revendiquer sa propre culture. Le Clézio a très bien tracé le cheminement d'une convergence des différentes cultures. La fugue de Gaspar ne reflète que cette nécessité d'aller à la rencontre de l'autre pour découvrir du nouveau pour s'enrichir. La communion de Gaspar avec les enfants nomades n'est qu'une invitation à la compréhension mutuelle : leur amitié est baptisée par le partage quotidien et les sacrifices et non pas par des valeurs conventionnelles qui vident l'être humain de sa propre nature. En effet, débarrassé de ses chaînes de la société civile, Gaspar a pu recouvrer sa vraie identité d'un terrien au-delà des frontières psychiques et langagières. L'impossibilité d'échanger dans une langue commune rapproche les enfants au lieu de les éloigner car cela ouvre le chemin au langage spontané et naturel qui exprime adéquatement les besoins réels des êtres à travers la transparence et l'innocence des enfants. Le Clézio fait valoir un langage 
universel dépassant les cultures et les identités : ce langage guidé par le silence et émanant de la nature elle-même, peut réunir non seulement les humains entre eux, mais aussi toutes les créatures vivantes. Savoir écouter le langage de la nature à travers ses éléments, conduit l'homme non seulement à se guider mais aussi à communiquer avec tout le cosmos qui est une réalité vivante et tangible par les sens et l'imagination.

C'est un grand chapitre que Le Clézio a essayé d'ouvrir afin de concilier les cultures en respectant la particularité de chacune. Cela a été nourri par ses propres expériences multiculturelles comme le confirme Özgüler (2016) «les déplacements multiples de l'écrivain à travers les trois grandes géographies culturelles (Europe, Amérique et Afrique) s'accompagnent aussi d'un voyage intérieur par le biais d'une écriture poétique faisant valoir l'autre, comme "le reflet de soi-même » et la diversité culturelle comme richesse et non comme fait de discrimination raciale ou de particularisme identitaire ». En effet, une magnifique entente a été construite entre Gaspar et les quatre enfants nomades. Mais cette entente a été cassée lorsque Gaspar avait essayé de toucher la question de survie qu'Abel avait abordée avec agressivité. Comment peut-on comprendre ce conflit qui avait surgi entre Abel et son compagnon ? Durant cette aventure à Genna, que Gaspar aimait, ils avaient vécu en harmonie en se défendant l'un l'autre et luttaient ensemble pour faire vivre tout le groupe mais la pression de la faim et la question de la survie s'était imposée comme une réalité plus forte que leur solidarité. Pour Gaspar, l'oiseau ibis était son ami, le roi qui pourrait donner des ordres pour que la nature garde toujours sa beauté et assure tous les éléments nécessaires à la vie heureuse. Pour Abel, la vie des bergers vient en première classe et il «se voit révéler la liberté du nomadisme" comme affirme Le Clézio (2008). Il aimait certainement l'ibis, mais il ne le préférait pas à sa sœur Khaf, à ses deux frères et au troupeau qui leur assurait le lait. Abel représentait la réalité tandis que Gaspar était guidé par l'idéal pur qui n'a pas pu se convertir complétement en réel.

Pour Le Clézio, il faut s'adapter aux échelles du temps et de l'espace en faisant un compromis entre les humains et la nature, mère de tous. L'affrontement final entre Gaspar et Abel sonne le glas d'un danger qui guette cet équilibre naturel et attire l'attention de l'homme sur un fait réel : la survie menacée des hommes et la nécessité de trouver des solutions qui pourraient adoucir le sort de la vie terrestre.

Gaspar qui fait irruption dans le monde harmonieux d'Abel ne seraitil pas l'un des descendants des Caïn dispersés sur la terre et écrasés par le fardeau des civilisations sédentaires, cloués au sol pour s'emprisonner davantage dans leur propre enfer civil coupé de la nature où l'artificiel et le virtuel remplacent l'univers réel, terre et ciel. Abel et le groupe restreint d'enfants-bergers vivant dans le désert ne seraient-ils pas les survivants 
mythiques d'un Abel pasteur nomade disparu à jamais depuis le temps biblique, comme pour souligner l'extinction d'une race en parfaite harmonie avec les éléments et avec leur divinité ? C'est un cri de l'auteur pour sauvegarder ces cultures en voie de disparition et menacées par l'incompréhension et la négligence. Cette escapade de Gaspar, selon le mode leclézien, est porteuse d'un message à l'humanité qui a oublié ses propres repères en s'éloignant de plus en plus à titre égal de sa propre nature et du cosmos qui est sa véritable identité.

\section{Références}

Augé, M. (1994). le sens des autres. Actualité de l'anthropologie. Paris: Fayard.

Ben Aïssa, Y. B. (2013). Altérité et marginalité dans les cuvres de JeanMarie Le Clézio et Amin Maalouf. Université Nantes Angers Le Mans et de l'Université de Manouba (L'unam), Thèse de Doctorat.

Boncenne, P. (1978). J.M.G. Le Clézio s'explique. Lire, 32.

Boulos, S. M. (1999). Chemins pour une approche poétique du monde. Le Roman selon J.M.G. Le Clézio. Museum Tusculanum Press, Kobenhavns Universitet.

Bouvet. (2012). Les paradoxes de l'altérité et la traversée des cultures dans l'œuvre de Le Clézio, publié dans J.M.G. Le Clézio dans la forêt des paradoxes,79-86. Harmattan, collection «Études transnationales, francophones et comparées».

Bouvet, R. (2010). le botaniste et la guérisseuse : figures de l'imaginaire végétal dans La Quarantaine de Le Clézio. Recherches sémiotiques, 30, 179-188. doi: 10.7202/1025932ar

Chanvallon, S. (2010). Anthropologie des relations de l'Homme à la Nature : la Nature vécue entre peur destructrice et communion intime. Université Rennes 2 ; Université Européenne de Bretagne. doi:https://tel.archives-ouvertes.fr/tel-00458244

Diouf, A. (2016). Déconstruction-reconstruction identitaire et poétique de l'altérité dans Le procès-verbal de J-M G. Le Clézio. Voix plurielles, 13(2), 150-162. 
Dufour, J. (2018). les romans de J.-M.G. Le Clézio : Rôle de l'écrivain contemporain dans la fondation d'une littérature mondiale considérée comme pratique. Université Sorbonne Nouvelle - Paris-3, Thèse de Doctorat.

Dutton, J. L. (1999). Le Chercheur d'or et d'ailleurs : Le Clézio sur le chemin de l'utopie (1963-1998),. University of Adelaide, Centre for European Studies . doi:http://hdl.handle.net/2440/19546

Holzberg, R. (1976). La Dialectique du silence dans l'œuvre de J.M.G. Le Clézio. Rutgers State Univ. of New Jersey-New Brunswick.

Holzberg, R. (1981). L'Eil du serpent : dialectique du silence dans l'œuvre de J.M.G Le Clézio. Sherbrooke: Naaman.

Le Clézio, J.-M. G. (1978). Mondo et autres histoires. Paris: Gallimard.

Le Clézio, J.-M. G. (1980). Désert. Paris: Gallimard.

Le Clézio, J.-M. G. (1985). Le chercheur d'or. Paris: Gallimard.

Le Clézio, J.-M. G. (2008). Peuple du ciel. Paris: Gillamard.

Manijashvili, I. (2014). le silence comme moyen de communication. Université d'Etat Iv. Djavakhishvili de Tbilissi, Géorgie, 149-156. Récupéré sur ojs.iliauni.edu.ge

Özgüler, C. (2016). Les Enjeux De L'Interculturel Dans L'Oeuvre Romanesque De J.-M.G. Le Clézıo,. Université Hacettepe. Institut des Sciences Sociales. Département de Langue et Littérature Française. Thèse de Doctorat. Récupéré sur http://hdl.handle.net/11655/3606

Roussel-Gillet, I. (2008). Les cartes du ciel à l'œuvre chez Le Clézio. Revue d'étude de dialogue texte-image (Textimage), $N^{\circ} 2$ Cartes et Plans, $1-17$.

Sayegh, M. (2020c). Mondo de J.M.G. Le Clézio : Épreuves du monde orphelin. European Scientific Journal (ESJ), 16(23), 56-78.

Sueza, M. J. (2009). Désert de Jean-Marie Gustave Le Clézio : analyse d'éléments descriptifs et interprétation écocritique. Çédille, revista de estudios franceses(5), 329-346.

Tritsmans, B. (2005). Figures du berger chez J.-M.G. Le Clézio et A. Dhôtel,. Nouvelles Etudes Francophones (NEF), 20(2), 57-68. 
Vartian, S. (2000). Désert et immensité intime chez J.M.G. Le Clézio. Figura, Centre de recherche sur le texte et l'imaginaire, 1, 27-47.

Vogl, M. B. (2005). Le Clézio en noir et blanc : La Photographie dans L'Africain. Nouvelles Études Francophones (NEF), 20(2), 79-86. 